\title{
Clinical and Experimental Psychology
}

\section{Verbal Exposure for Irrational Fears: New Directions in Research and Applications}

\section{Rakesh Kumar*}

Institute of Mental Health and Hospital, Agra, Uttar Pradesh, India

\section{Introduction}

Exposure therapies are proven to be effective in irrational fears such as seen in Obsessive-Compulsive Disorder (OCD) and phobia [1-5]. Typically, an individual with irrational fears is exposed to the feared stimuli in imagination and/or in vivo along with prevention of avoidance response. The exposure in this manner initially leads to an increase in fear reaction which gradually begins to decline. The repeated exposure in this manner leads to habituation or elimination of fear reaction. For instance, in an individual with fear of lizards, imaginal exposure would involve repeated holding of imagery of lizard including feared consequences in the content of consciousness. For in vivo exposure, the patient would be encouraged being repeatedly in proximity of actual lizard and imagining feared consequences without avoidance response. Multiple theories have been put forth to explain the mechanisms of action involved in exposure therapies. Rachman [6] and Foa and McNally [7] proposed Emotional Processing Theory to explain the mechanisms of action in exposure therapy. Craske et al. [8] and Labordaan Miller [9] offered Inhibitory Learning Theory for the same.

\section{Exposure Therapies}

At least four components are present in conditions of phobia and OCD. (1) Stimuli, the exposure to which elicits fear reaction. Because of stimulus generalization a number of stimuli become associated with fear reaction. For example, in case of lizard phobia, lizards, pictures of lizards, videos of lizards, mental imagery of lizard, size, numbers, position and movements of lizards, talking and hearing about lizard, any animal that bears a similarity with lizard; all can potentially elicit fear reaction. (2) Threat perception, exposure to feared stimuli trigger a threat perception which may involve a threat to life, stability, social status, illness or loss of any kind. In above case, it may get reflected in catastrophizing thoughts of harm to the body and existence such as if it falls on the body, it can cause skin disease, fainting, poisoning and so on. (3) Sympathetic arousal. (4) Safety behaviors usually in the form of avoidance, elimination of feared stimuli from the environment or compulsive acts. These behaviors are aimed at to ensure safety against potential threat. An individual with lizard phobia can take safety measures like removal of lizard from home, sealing windows and ventilators to prevent entry of lizards, installing glasses on the wall so that lizard does not creep on walls, if lizard is present, asking someone to throw it out and the like.

In contamination $\mathrm{OCD}$, the four components could readily be discerned as (1) dirty objects triggering obsession of contamination as stimuli (2) threat perception in the form of spreading contamination, catching illness and polluting religious objects which could cause misfortune (3) sympathetic arousal (4) compulsions and rituals of cleaning and washing; also the avoidance of dirty objects as far as possible.

Stimuli are simply feared cues that trigger threat perception. In a cognitive framework, threat perception is the primary determinant of irrational fears. The aim of treatment is (a) to disconfirm threat perception associated with feared stimuli (Emotional Processing Theory) and (b) learning competing safety associations that the thoughts of threat perception are not dangerous (Inhibitory Learning Theory). The goal is neither removal of feared stimuli from the environment nor elimination of fear reaction or threat perception from being of the individual. When irrational threat perception ceases to get triggered by stimuli or feared stimuli trigger competing safety thoughts, the problem can be considered as resolved which get manifested in emotional and behavioral non-reactivity to previously feared stimuli.

In a time reference perspective, threat perception corresponds to future time frame. It involves probabilities of causing harm of some kind in future time. Human beings use three time reference (a) past (b) present and (c) future. Threat perception capitalizes on future and remains devoid of past and present time frame. For example, lizard has not yet caused harm (past time reference) or if caused any harm in the past, then it can do so in future also beyond normal probability (future time reference); lizard is not causing harm right now (present time reference) but if lizard continues to be present in immediate environment, then it can potentially cause harm (future time reference).

It could be hypothesized that three time references do not have identical neural correlates. That means, past, present and future time references have distinct neural pathways. Feared stimuli and threat perception capitalize on neural pathways involved in processing of future time reference. If we can find methods of shifting of the processing of threat perception triggered by feared stimuli from future reference related neural pathways to past or present related neural pathways; it can then exploit inbuilt neuroplasticity and resolve the conditions of irrational fears [10].

One method of achieving this shift of neural pathways is through a combination of verbal and imaginal exposure. Under conditions of phobia and OCD, an individual keeps on imagining threatening probabilities and his/her sub-vocal speech contains the future time reference. This covert scenario can be altered by prescribing a change in time reference from future to past or present both in imagination and at vocal/sub-vocal level. For example, for lizard phobia, the actual procedure would involve asking the individual to imagine a lizard and verbally repeat that lizard has fallen over me and actually caused me skin disease. Repeat, repeat and repeat. This way of doing verbal exposure

*Corresponding author: Kumar R, Senior Clinical Psychologist, Institute of Menta Health and Hospital, Mathura Road, Billochpura, Agra 282002, Uttar Pradesh, India, Tel: + 09219770137; E-mail: jain.imhh@gmail.com

Received: July 01, 2017; Accepted: July 06, 2017; Published: July 13, 2017

Citation: Kumar R (2017) Verbal Exposure for Irrational Fears: New Directions in Research and Applications. Clin Exp Psychol 3: 157. doi: 10.4172/24712701.1000157

Copyright: @ 2017 Kumar R. This is an open-access article distributed under the terms of the Creative Commons Attribution License, which permits unrestricted use, distribution, and reproduction in any medium, provided the original author and source are credited. 
Citation: Kumar R (2017) Verbal Exposure for Irrational Fears: New Directions in Research and Applications. Clin Exp Psychol 3: 157. doi: 10.4172/2471-2701.1000157

is labeled as Cognitive Drill [11]. At the onset of this repetition, sympathetic arousal will shoot up from medium to high. If it gets very high, a pause of 30 seconds or more could be given. The continued imagination and verbal repetitions with changed time frame will lead to resolution of sympathetic arousal and fear reaction at behavioral level within a couple of minutes. At cognitive level it will lead to 'empirical realization' of falsification in the threatening probability triggered by otherwise neutral stimuli and formation of safety associations that the thoughts of threat perception are not dangerous. At neural level there might be a shift in the neural pathways triggered by fear provoking stimuli. Verbal and imaginal exposure would provide an opportunity for real-time scanning of neural activities for changes in the brain in addition to mapping changes in areas in the brain due to exposure. Verbal and Imaginal exposure could be sufficient to resolve irrational fears. However, in other cases, this exposure can incorporate exposure to actual stimuli coupled with verbal repetitions of time-reference modified statements.

Verbal exposure is based on empirically supported principles of exposure treatment. It is a novel procedure in the family of exposure therapies which has huge research potentials. A few case studies have been shared so far which are based on verbal exposure. Specific phobia [12]; Agoraphobia with panic disorder [13]; OCD [11]. In addition to further case studies, future research will focus on delineation of neural correlates of verbal exposure, randomized controlled trials and comparative efficacy studies of verbal exposure vis-à-vis existing methods of exposure therapies.

\section{References}

1. Abramowitz JS (1996) Variants of exposure and response prevention in the treatment of obsessive-compulsive disorder: A meta-analysis. Behavior Therapy 27: 583-600
2. Abramowitz JS (1997) Effectiveness of psychological and pharmacologica treatments for obsessive-compulsive disorder: A quantitative review. J Consult Clin Psychol 65: 44-52.

3. Abramowitz JS, Franklin ME, Foa EB (2002) Empirical status of cognitivebehavioral therapy for obsessive-compulsive disorder: A meta-analytic review. Romanian Journal of Cognitive and Behavioral Psychotherapies 2: 89-104.

4. Eddy K, Dutra L, Bradley R, Weston D (2004) A multidimensional meta-analysis of psychotherapy and pharmacotherapy for obsessive-compulsive disorder. Clin Psychol Rev 24: 1011-1030.

5. Franklin ME, Abramowitz JS, Foa EB, Kozak MJ, Levitt JT (2000) Effectiveness of exposure and ritual prevention for obsessive-compulsive disorder: Randomized compared with nonrandomized samples. J Consult Clin Psychol 68: 594-602.

6. Rachman S (1980) Emotional processing. Behav Res Ther 18: 51-60.

7. Foa EB, McNally RJ (1996) Mechanisms of change in exposure therapy

8. Craske MG, Kitcanski K, Zelokowsky M, Mystkowski J, Chowdhury N, et al (2008) Optimizing inhibitory learning during exposure therapy. Behav Res Ther 46: 5-27.

9. Laborda MA, Miller RR (2012) Reactivated memories compete for expression after Pavolovian extinction. Behav Processes 90: 20-27.

10. Månsson KNT, Salami A, Frick A, Carlbring P, Andersson G, et al. (2016) Neuroplasticity in response to cognitive behavior therapy for social anxiety disorder. Transl Psychiatry 6: e727.

11. Kumar R, Sameer A, Singh B (2012) Preliminary test of cognitive drill as an intervention. Indian J Clin Psychol 39: 67-74.

12. Arya B, Verma S, Kumar R (2017) Efficacy of cognitive drill therapy in treatment of specific phobia. SIS Journal of Projective Psychology and Mental Health 24: 48-51.

13. Dwivedi S, Kumar R (2015) Efficacy of cognitive drill therapy in agoraphobia with panic disorder: A case study. SIS Journal of Projective Psychology and Mental Health 22: 139-146. 